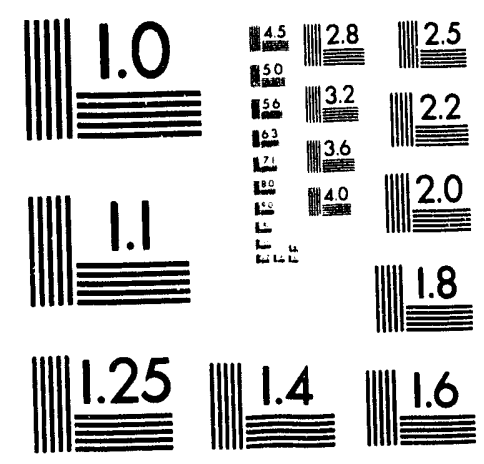



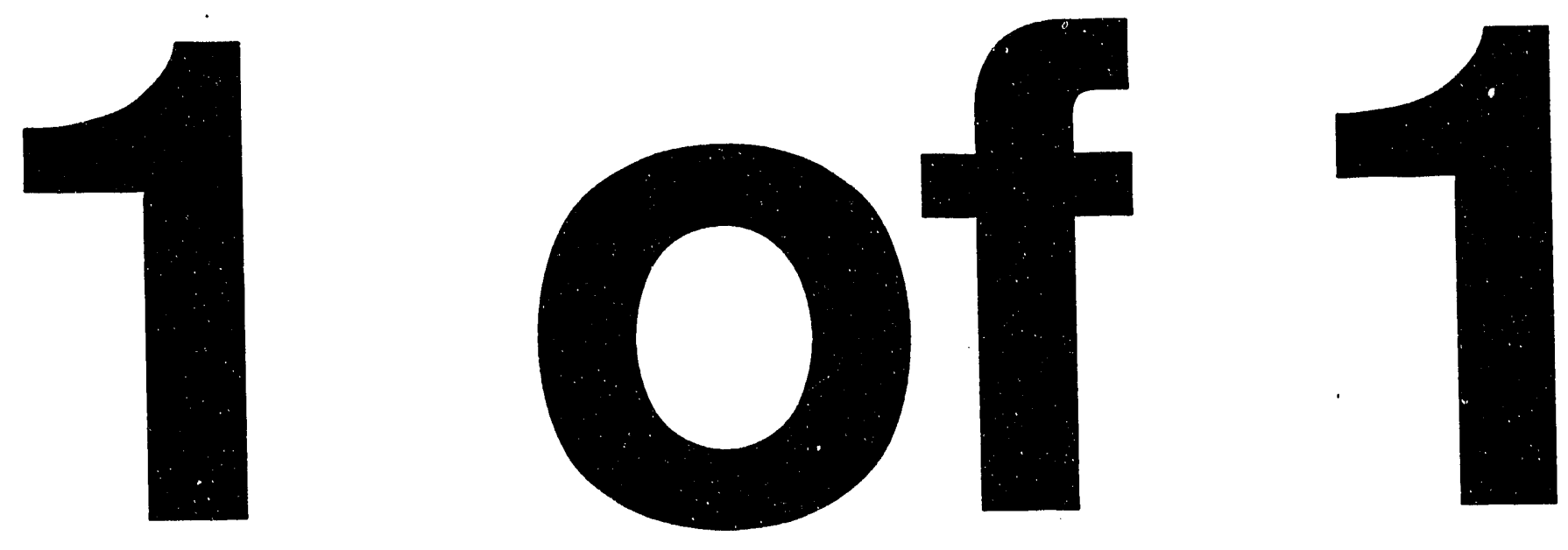


\title{
Wire Scanner Data Analysis for the SSC Linac Emittance Measurement*
}

\author{
C. Yao, J. Hurd, and J. Sage \\ Superconducting Super Collider Laboratory ${ }^{\dagger}$ \\ 2550 Beckleymeade Ave. \\ Dallas, TX 75237
}

July 1993

"Presented at the 1993 IEEE Particle Accelerator Conference on May 17-20, Washington, D.C.

†Operated by the Universities Research Association, Inc., for the U.S. Department of Energy under Contract No. DE-AC35-89ER40486. 


\title{
Wire Scanner Data Analysis for the SSC Linac Emittance Measurement
}

\author{
C. Y. Yao, J. W. Hurd, and J. Sage \\ Superconducting Super Collider Laboratory* \\ 2550 Beckleymeade Ave., Dallas, TX 75237 USA
}

\begin{abstract}
The wire scanners are designed in the SSC Linac for measurement of beam emittance at various locations. In order to obtain beam parameters from the scan signal, a data analysis program was developed that considers the problems of noise reduction, machine modeling, parameter fitting, and correction. This program is intended as a tool for Linac commissioning and also as a part of the Linac control program. Some of the results from commissioning runs are presented.
\end{abstract}

\section{INTRODUCTION}

The SSC linear accelerator is a $600 \mathrm{MeV} \mathrm{H}^{-}$Linac including a $35 \mathrm{KeV}$ ion source, a low energy beam transport section, a $2.5 \mathrm{MeV}$ RFQ acceleration section, a $70 \mathrm{MeV}$ Drift Tube Linac, and the final $600 \mathrm{MeV}$ Coupled Cavity Linac. The required beam emittance is $0.3 \pi \mathrm{mm}$-mrad in both $\mathrm{X}$ and $\mathrm{Y}$ directions.

In order to achieve the desired goal, emittance growth control is important not only at the design and construction stage, but also during commissioning and operation.

The wire scanner system is one of the main emittance measurement methods for the SSC Linac. A total of 18 wire scanners are designed in the Linac and transport line. They are located at different stages of beam acceleration and will be used for on-line beam matching and optimization.

\section{WIRE SCANNER FOR SSC LINAC}

A schematic of the wire scanner is shown in Figure 1. The design is similar to the SLAC SLC wire scanner [4], [5].

The wire is made of gold plated graphite fiber with a diameter of $33 \mu \mathrm{m}$. The frame is made of ceramic material plated for wire mounting. Three wires are mounted in such a way that the wires cross the beam in $\mathrm{X}, \mathrm{Y}$, and at a $45^{\circ}$ orientations in a complete scan.

The wire frame is driven by a stepping motor.

When the wires are struck by the beam, both secondary emission and forward scattering is produced, which is reflected in the signals on the wire. The wire signals are brought to a sample amplifier and a 12-bit wave form digitizer to convert into digital wave form data. Wire signals are sampled by the amplifiers at about a $5 \mathrm{MHz}$ rate to ensure adequate bandwidth for observation of longitudinal macro bunch structure.

*Operated by the Universities Research Association, Inc., for the U.S. Department of Energy under Contract No. DE-AC35-89ER40486.

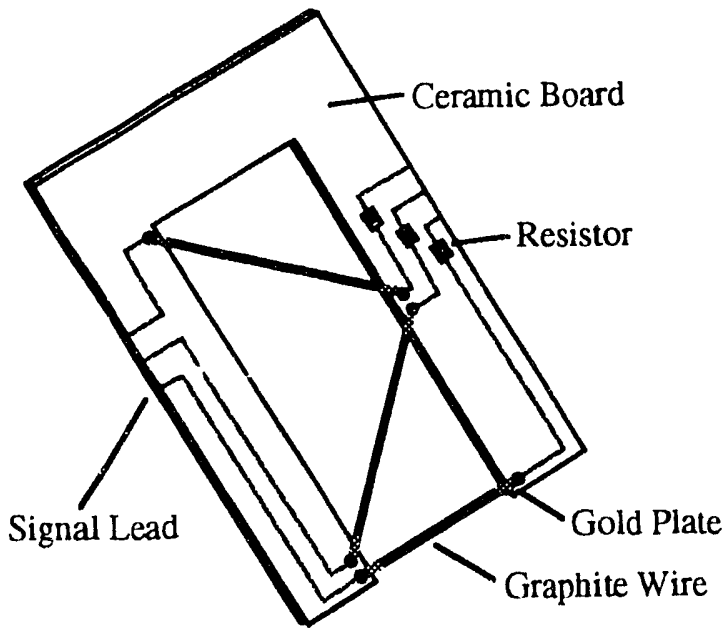

Figure 1. Schematic of SSC wire scanner.

\section{DATA ACQUISITION SYSTEM}

Wire scanner data acquisition is performed by the Linac control system, which is a distributed control system consisting of SUN workstations, network servers, and local control systems (IOC). Each IOC is a VME system running VxWorks operating system and EPICS.

Wire scanner signals are connected to a VME wave form digitizer module through a preamplifier and a multipurpose interface card. Wire motion is driven by a SMC controller board, and the position is sensed by a LVDT. A complete scan contains about 200 position data scans, each with 500 sampling points.

Data acquired by the IOC are read by a SUN workstation through Channel Access. The data is then sent to an Oracle database on the network, which is accessible to all the workstations.

\section{DATA ANALYSIS PROGRAM}

The wire scanner data analysis program is designed for commissioning and operation of the Linac. The main requirements are effective and practical algorithm, friendly user interface, ease of operation, and access. It is designed as an $\mathrm{X} /$ Motif application and can be run on any workstation on the Linac control system network.

It mainly consists of four functional parts: user interface, wire data display, r.m.s. beam width calculation, and emittance fitting.

\section{A. User Interface}

A main window with menu button and text window provides program control and message display. A popup 
window provides various plot functions. Display options are selected by selection buttons.

\section{B. Wire Data Display}

The main function of the wire data display is to provide the operator with a direct view of the acquired data. Noises, hardware failure, and other possible errors will cause false data. By careful study of the original data, some problems can be diagnosed and treated.

The display program provides several views of the data. A longitudinal view gives the data variation with time, which reveals the beam distribution along the longitudinal direction and signal spectrum generated by hardware or noises. Threedimensional view and single curve step through options are provided to allow observation with different respective. Background signal subtraction, filtering, averaging, and smoothing are provided to aid diagnosis. Figure 2 shows a typical display.

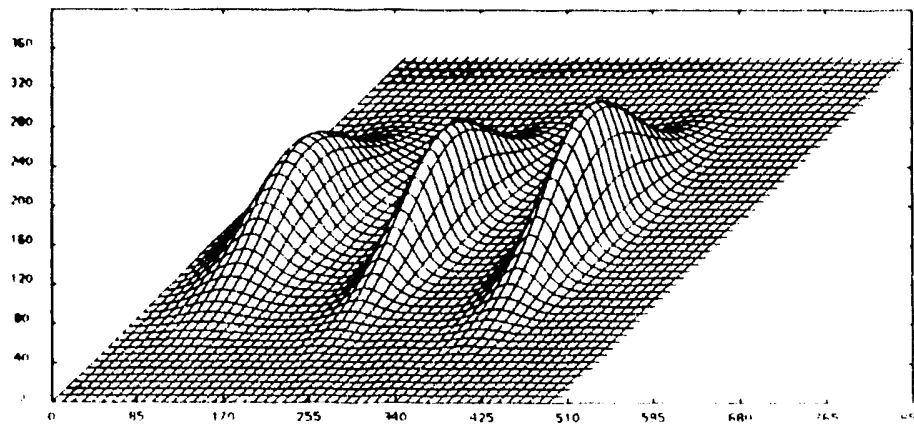

Figure 2. Wire data display.

Data are also displayed in text format for careful review, and a data editor is also incorporated to allow the operator to eliminate outliers due to interferences or hardware failure.

\section{R.M.S. Width Calculation}

It was shown that to first order, which this program is intended for, beam behavior could be well represented by an equivalent beam distribution with the same r.m.s. width. We choose Gaussian distribution to fit the beam profile. The transverse beam profile is defined by:

$$
\varrho_{2}=\int \varrho_{4} e^{-x^{T} \cdot \sigma^{-1} x_{d x^{\prime}} d y^{\prime}},
$$

where $\mathrm{X}$ is four-dimension phase space vector, $\sigma$ is transverse emittance matrix, $\varrho_{2}, \varrho_{4}$ are real space and phase space beam distribution.

For single amplifier configuration, the beam signal is the sum of three signals from each of the wires. If the wire separations are large enough, the signals are easily separated. When the size of beam profile is comparable to the wire separations, signals become overlapped and a signal separation routine is developed.

Signal center and width are calculated through first and second moments calculation. A least square fitting routine is then used to do a final fitting. The program outputs position of the beam, signal amplitude, and r.m.s. beam width as well as bunch tilt angle. Fitting results are also graphically displayed. Figure 3 is a display of the fitting result.

center $x=250$ center $y=-50$ height $=0.63$
sigma $r 1=0.002502,519$ ma2 $2=0.001373$, sigm

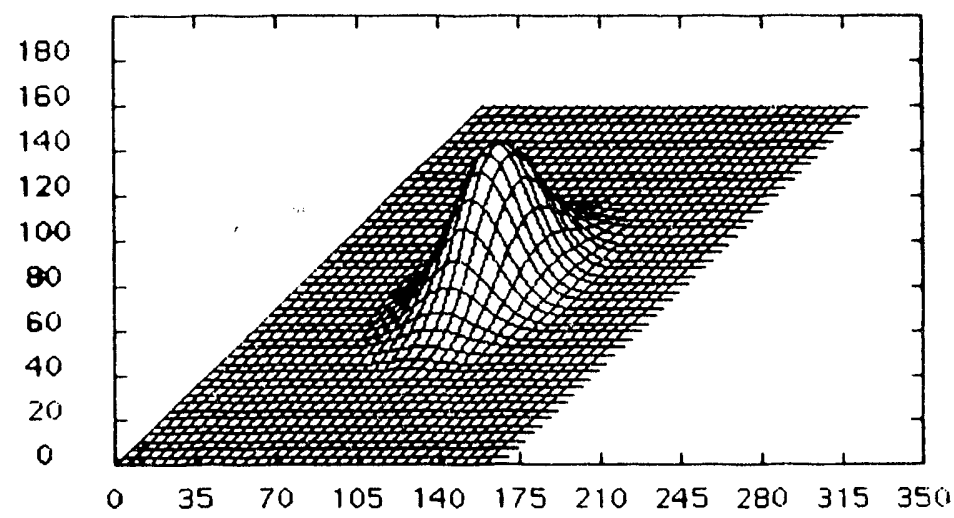

Figure 3. Display of fitted beam profile.

\section{Emittance Fitting and Matching}

Several methods can be used to measure transverse beam emittance. Here we only describe the fixed optics method, which is also suitable for on-line matching correction [1], [2], [3].

For a fully coupled beam, $\sigma$-matrix can be reconstructed by measurement of beam profile at 4 locations. For the decoupled case, three measurement are adequate. In the following description the decoupled case is assumed.

Due to errors in the width measurement and errors in machine parameter and modeling, directly solving the linear equations may not give good results. We use a least square minimization to fit the $\sigma$-matrix, which presents the r.m.s. emittance and Twiss parameters of the beam.

A series of wire scanners are used to reconstruct the r.m.s emittance fitting and matching. Figure 4 outlines the algorithm. The iteration is needed because of the space charge effect at low beam energy.

First the $\sigma$-matrix are calculated using first-order transformation, which does not include space charge effects. Based on this $\sigma$-matrix new transformation matrices are generated, which are used to make another $\sigma$-matrix fitting. This process is iterated until a convergence is reached.

The least square fitting process is described briefly here. Let $\sigma(0)$ denote the desired beam $\sigma$-matrix, $\sigma(\mathrm{n})$ for $\sigma$-matrices at wire scanner $n$ and $R(n)$ for the transformation matrix from the point where $\sigma(0)$ is associated to the nth wire scanner location. The relation between $\sigma(0)$ and $\sigma(n)$ is given by:

$$
\sigma(n)=R(n) \sigma(0) R^{T}(n) .
$$

The measured r.m.s. radius, $x(\mathrm{n})$, of the beam is related to the $\sigma$-matrix elements by

$$
x_{m}(n) \propto \sqrt{\sigma_{11 m}(n)},
$$

where subscript $m$ denotes measured values. 
For each wire scanner $n$, we have

$$
\sigma_{11}(n)=\sum_{i, j=1}^{2} R_{1 i}(n) \sigma_{i j}(n) R_{1, j}(n) .
$$

The total error is minimized for

$$
\chi^{2}=\sum_{n=1}^{N}\left[\sigma_{11 m}(n)-\sum_{i, j=1}^{2} R_{1 i}(n) \sigma_{i j}(0) R_{1 j}(n)\right]^{2} .
$$

The least square minimization criterion is satisfied by solving the three equations given by

$$
\sum_{n=1}^{N}\left[R_{1 k}(n) \sigma_{11 m}(n) R_{11}(n)-R_{1 k}(n) R_{11}(n) \sum_{i j=1}^{2} R_{1 i}(n) \sigma_{j i j}(0) R_{1 j}(n)\right]=0
$$

for $k l=11,12,22$. This gives the element of $\sigma(0)$.

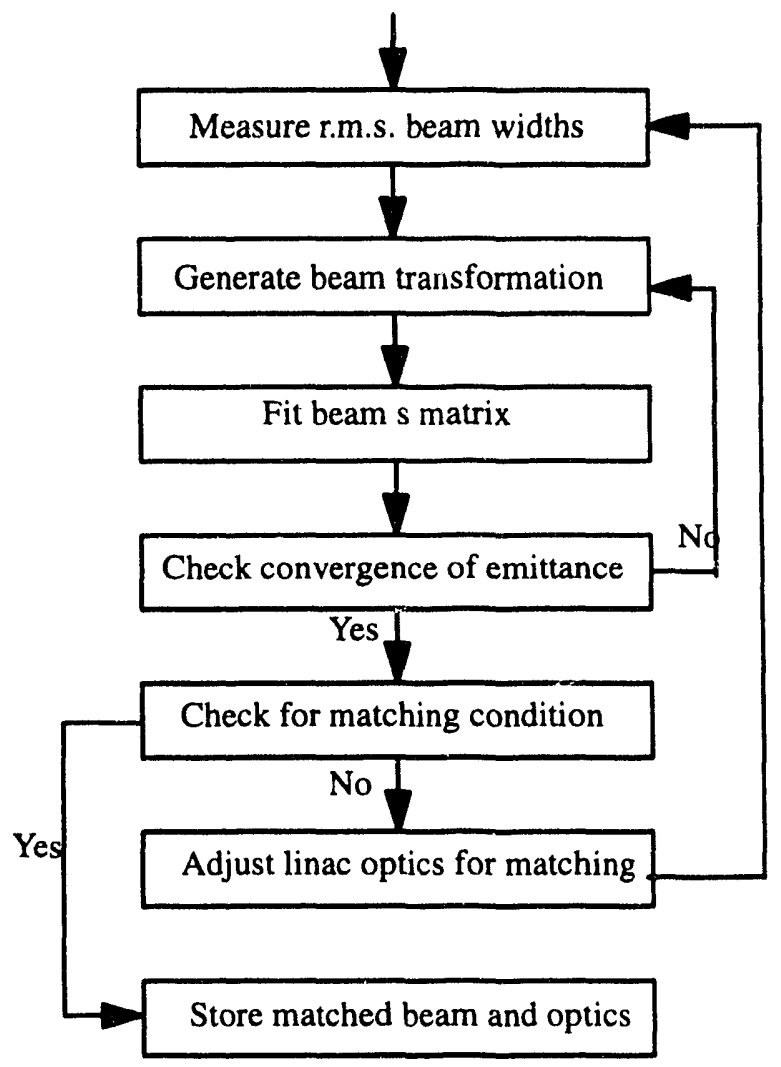

Figure 4. Fitting and matching algorithm.

\section{REFERENCES}

K.L. Brown et al., "Transport - A Computer Program for Designing Charged Particle Beam Transport Systems," Stanford Linear Accelerator Center report SLAC-91, May 1977.

G. Swain et al. "Generating Catalogs of Transverse Matching Solutions," Proc. of the 1989 IEEE Particle Accelerator Conference.

[3] K.R. Crandall, "Trace: An Interactive Beam-Transport Program," Los Alamos Scientific Laboratory Report LA-5332, October 1973.

[4] A. Burns et al., "Wire Scanner News from the CERN-SPS," Proc. of 1989 IEEE Particle Accelerator Conference.

[5] M.C. Ross et al., "Wire Scanners for Beam Size and Emittance Measurement at the SLC," Proc. of 1991 IEEE Particle Accelerator Conference. 

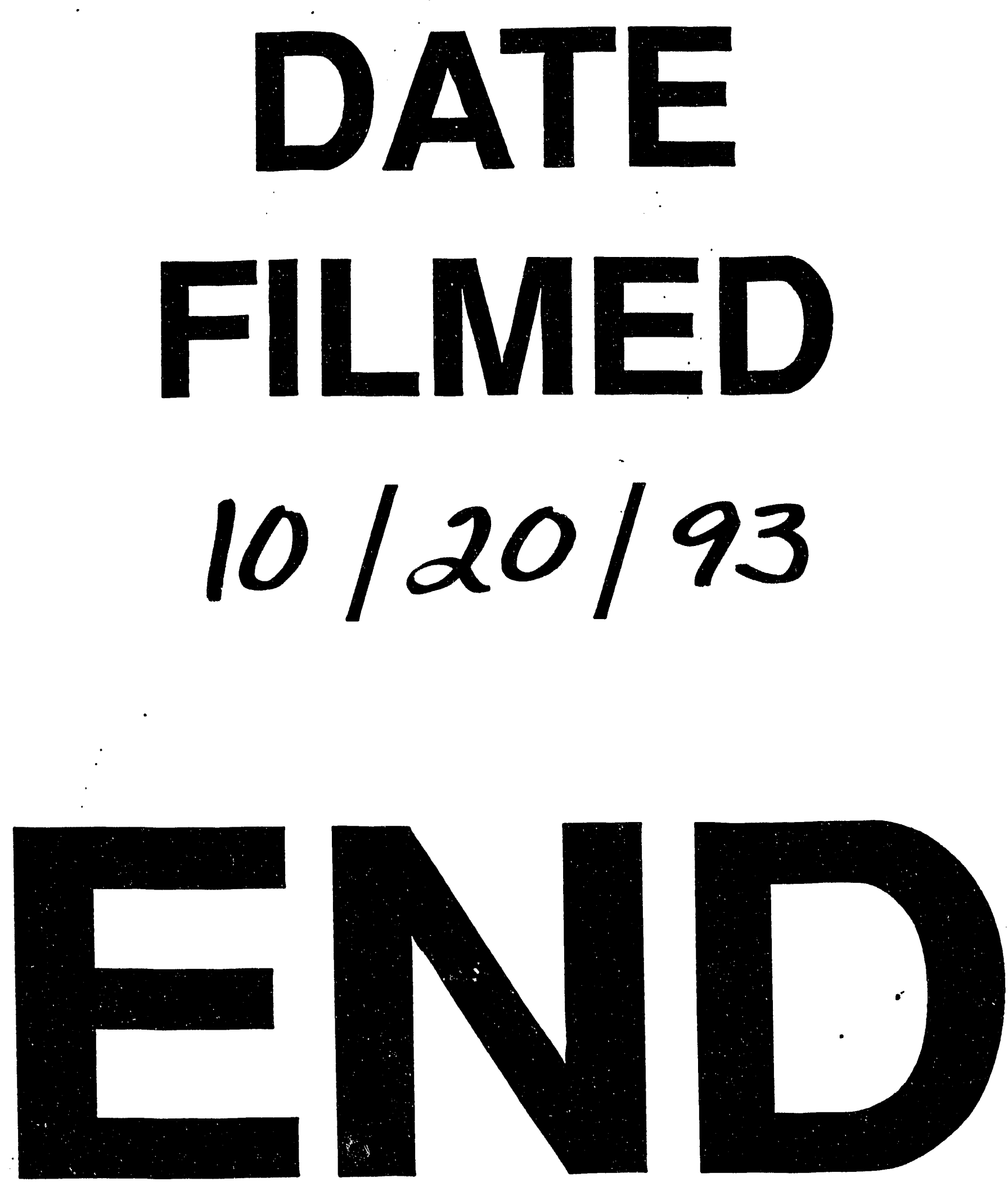
